\section{La faim du monde}

Nasser, Éditions Balland, 2019, 218 pp., ISBN : 9782940632046

C'est un petit ouvrage de poche bien singulier que La faim du monde, rédigé par Nasser, consultant auprès d'agences et de programmes des Nations Unies, tels que le Fonds international de développement agricole (FIDA) et la Food and Agriculture Organisation (FAO). Tout simplement parce qu'il se propose de trouver la voie vers la fin de la faim à l'échelle de toute la planète. Pour cela, l'auteur invite à un voyage à trois dimensions. Dans la première, il présente les causes qui mènent inéluctablement à la fin de la vie humaine sur la Terre, si rien n'est fait pour y remédier. Il revient ainsi sur les grands changements qui ont affecté les pratiques agricoles et les perturbations des écosystèmes naturels depuis la fin de la deuxième guerre mondiale. Tout y passe: les pollutions des sols, les usages insensés des pesticides, le déclin de l'élevage pastoral et l'irruption des productions animales intensives à base d'aliments concentrés, la surpêche, la déforestation accélérée et le braconnage, et leurs conséquences désastreuses. Sans omettre les pressions insoutenables sur les ressources, notamment l'accaparement des meilleures terres, ainsi que le travail pénible et mal rémunéré des plus faibles, comme les enfants.

Dans la deuxième dimension de son raisonnement, l'auteur commence par énumérer les raisons et les conséquences de la faim dans le monde et ses différentes manifestations. Il rappelle que près d'un être humain sur neuf souffre de la faim, et que ce sont surtout des paysans pauvres de pays en développement qui en sont les premières victimes. Il présente aussi les causes de la faim, notamment naturelles (les inondations, les tremblements de terre, etc.), mais aussi politiques, comme les conflits armés qui détruisent les infrastructures, les embargos, etc. Il dépeint ensuite les conséquences de la faim, principalement les retards de croissance des enfants et les dysfonctionnements de leurs facultés cognitives. Puis, il clôt son propos relatifà la faim dans le monde par un vaste tour d'horizon des acteurs impliqués, gouvernementaux et non gouvernementaux. L'auteur évoque au final les nécessaires principes de bonne gouvernance, aussi bien de la part des donateurs que des récipiendaires de l'aide, pour espérer un jour s'affranchir définitivement de la faim et de ses conséquences désastreuses.

La troisième et dernière partie de l'ouvrage s'intitule « la fin de la faim ». L'auteur y adopte un ton plus agressif, n'hésitant pas à comparer la faim à une « destruction massive d'êtres humains » qui concourt aussi à l'élimination de l'agriculture paysanne vivrière. Il se lance ensuite dans longue diatribe contre les responsables de la faim, dont la finance internationale, bras armé du libéralisme économique qui inonde les marchés des pays en développement de denrées alimentaires subventionnées, détruisant leurs systèmes agricoles locaux. Il cite aussi les multinationales qui ont privatisé le vivant, rendant caduques les velléités de sauvegarde des semences paysannes multiséculaires. Puis, il enchaîne par une énumération de solutions possibles pour éradiquer la faim, avec en priorité la réhabilitation du mot d'ordre de la souveraineté alimentaire des nations. Selon l'auteur, cela doit se faire dans un projet de communication continue auprès des populations les plus concernées par la faim, pour les informer de leur droit au développement, dans un esprit participatif. En outre, l'auteur insiste sur l'idée de mettre fin au pillage des terres agricoles et au gaspillage alimentaire, véritables fléaux qu'il faut à tout prix éviter. À cet égard, les femmes ont indéniablement un rôle important à jouer, tant leur contribution à la production des denrées agricoles et à leur transformation est fondamentale. Elles sont malheureusement souvent les plus guettées par la pauvreté, un des principaux déterminants de la faim. Pour en sortir, l'auteur revient sur la nécessité de la promotion d'une agriculture respectueuse de son environnement physique et même humain, notamment par la généralisation des principes de l'agroécologie, qui font appel aux savoirs locaux et aux circuits courts.

En conclusion, l'auteur se veut optimiste, malgré les augmentations des inégalités et de l'affermissement constaté de la mainmise de la haute finance sur les affaires du monde. Il se plaît à croire que l'objectif de l'éradication de la faim à l'horizon 2030 (Faim Zéro) annoncé par les Nations Unies est encore possible, si les bonnes volontés de tous se conjuguent. Espérons que son appel sera entendu.

Au-delà de la conclusion, un ensemble d'exemples d'interventions réussies sur le terrain sont présentés. Il s'agit d'expériences de développement agricole dans des pays d'Afrique et du Moyen-Orient, ayant concrètement permis à des populations d'améliorer leur sort quotidien, par la promotion de techniques durables de production ou de pêche ou par la sauvegarde d'écosystèmes naturels menacés.

Très abondamment documenté à l'aide de notes de bas de pages pertinentes que l'auteur utilise tout le long de son ouvrage pour convaincre le lecteur et le faire adhérer à son propos, ce livre de poche se laisse lire très facilement, tant par son style pétillant que par l'importance des thèmes qu'il traite. Il constitue ainsi un plaidoyer vibrant sur la nécessité de vaincre la faim et de changer de modèle de développement agricole.

Mohamed Taher Sraïri
Institut Agronomique et Vétérinaire Hassan II, BP 6202, 1, rue
Allal Al Fassi Madinate Al Irfane, 10101 Rabat, Maroc
* Auteur de correspondance: mt.srairi@iav. ac.ma

